\title{
40 Jahre erlebte Augenheilkunde
}

\section{Ein Rückblick auf die I. Univ.-Augenklinik (Wien) und die Augenabteilung des Donauspitals (SMZOst)}

\section{T. Michael Radda}

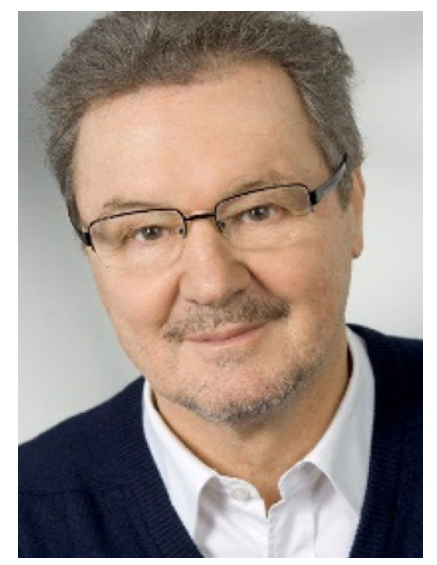

Ich begann meine ärztliche Tätigkeit am 01.07.1976 an der 1. Universitäts-Augenklinik (Prof. Deutsch) und wollte ursprünglich Internist werden. Da mich die Augenheilkunde sehr interessierte, hatte ich mich vor Abschluss meines Medizinstudiums auch an der I. Univ.-Augenklinik beworben.

Im Dezember 1977 rief mich Herr Univ. Prof. Dr. Heinz Schenk, damals Personalchef, an und bot mir eine Ausbildungsstelle als Assistenzarzt an. So begann ich meine Tätigkeit an der Univ.-Augenklinik mit 01.02.1978. Herr Univ. Prof. Karl Hruby leitete damals die I. Augenklinik, die sich im 4. und 5. Hof des „Alten AKH“ befand, und Univ.-Prof. Hans Slezak leitete die II. Augenklinik, deren Eingang sich in der Alser Straße befand.

Als ich in die I. Univ.-Augenklinik eintrat, waren die habilitierten Oberärzte Doz. Gittler, Prof. Wolfgang Funder und Doz. Schwab. Der spätere Klinikvorstand, Heinz Freyler, stand knapp vor seiner Habilitation,

Prim. Univ.-Prof. Dr. T. M. Radda ( $\square)$

Sieveringerstr. 18/6, 1190 Wien, Österreich m.radda@aon.at und auch die anerkannte Strabologin Elfriede Stangler-Zuschrott war zwar Oberärztin, aber noch nicht habilitiert.

Die Einfahrt zu den Höfen im „Alten AKH“ wurde von Portieren bewacht, und ich habe bald erkannt, dass man sich durch ein kleines „Bakschisch“ einen Dauerparkplatz für sein Auto sichern konnte. Auch die Patienten kamen so zu ihren Parkplätzen. Das hatte zur Folge, dass die Tätigkeit als Portier im „Alten AKH“ sehr begehrt war, man aber nach 8 Uhr Früh keinen Parkplatz mehr bekam.

Die Stationen bestanden aus großen Sälen mit ca. 20 Betten in einem Raum, und daneben gab es 1 bis 2 kleinere Zimmer für die Sonderklassepatienten. Die einzelnen Stationen waren räumlich voneinander getrennt, und die Stationsleiter hatten mehr oder weniger ein "Miniprimariat“ inne.

Die Station 19 befand sich im 5. Hof im Erdgeschoss und wurde von Prof. Schenk geführt.

Frau Prof. Stangler-Zuschrott (damals noch nicht habilitiert) hatte auf dieser Station ihre strabologischen Patienten liegen. Auf der Station 19 befand sich auch ein Kinderzimmer.

Die Station 59 war die Chefstation von Prof. Hruby. Sie befand sich im 1 . Stock und bestand aus 6 Ein- bis Dreibettzimmern. Das Zimmer 7 (6 Betten) wurde von OA Dr. Hans Gnad betreut und lag am Ende eines langen Ganges. Prof. Hruby hatte einen ausgezeichneten Ruf in Griechenland, und mindestens $50 \%$ seiner Patienten kamen von dort. Ich durfte am Beginn meiner Tätigkeit 1 Semester lang an der Chefstation arbeiten, und es war sehr wichtig, einige griechische Worte $\mathrm{zu}$ beherrschen. Bei der Sehprobe z. B. musste man natürlich alle Ziffern auf Griechisch bezeichnen können. Auch den Satz „Bitte die Augen öffnen“ und die Frage „Haben Sie Schmerzen?“ beherrschte ich bald auf Griechisch. 
Die Station 58 und 57b waren auch im 1. Stock lokalisiert. Die Station 58 wurde von Dozent Freyler und die Station 57b von Prof. Funder geführt.

Bei der Kataraktoperation wurde damals der Augapfel am Limbus über $160^{\circ}$ aufgeschnitten und die Linse in toto mit dem Kryostift entbunden. Vorher wurde ein Muskelfaden unter den M. rectus superior gelegt. Gefürchtet waren intumeszente Katarakte, da häufig die Kapsel riss und Rindenreste aus der Vorderkammer gespült werden mussten. Natürlich gab es gelegentlich auch Glaskörperverluste. Ein peripheres Kolobom wurde obligat bei 12 Uhr angelegt. Präoperativ wurde nach der Retrobulbäranästhesie auch noch eine Akinese injiziert und auch noch eine Okulopression angelegt, um den Augendruck zu senken.

In den ersten Monaten meiner Tätigkeit an der I. Univ.-Augenklinik sah ich sogar noch, wie Dozent Gittler eine Kataraktoperation mit dem „Graeve-Messer“ operierte. Dabei wird das Auge am Limbus von temporal von 3 nach $9 \mathrm{Uhr}$ durchstochen und das Messer nach 12 Uhr durchgezogen.

Damals wurden noch keine Linsen implantiert. Die Aphakiekorrektur wurde mit der „Star-Brille“ oder Kontaktlinsen durchgeführt.

Die Augenambulanz wurde von Dozent Schwab gemeinsam mit Dozent Gittler geführt.

Die Assistenzärzte, die kurze Zeit vor mir an die Klinik gekommen waren, waren Dr. Paul Drobec, Dr. Ilse Scheimbauer und Dr. Brigitte Riss. Die Atmosphäre an der Augenklinik war nicht so kompetitiv wie an der internen Klinik (Prof. Deutsch), sondern eher locker und freundschaftlich.

Die Sekretärinnen, Frau Schauersberger und Frau Unteregger, waren wichtige Respektspersonen.

Schon bald nach meinem Eintritt in die Klinik wurde von Oberarzt Gnad mit Pars-plana-Vitrektomie mit dem „Klöti-Gerät“ begonnen [1]. Anfang der 80er-Jahre war die Pars-plana-Vitrektomie an der I. Univ.-Augenklinik schon fast eine Routineoperation, an der II. Augenklinik war sie leider noch nicht etabliert.

Die II. Univ.-Augenklinik war auf „Elektrophysiologie" spezialisiert, womit sich die I. Augenklinik nicht beschäftigte.

Nun erlitt im Jahr 1979 der legendäre Bundeskanzler Dr. Bruno Kreisky einen Zentralvenenverschluss auf einem Auge. Sein behandelnder Augenarzt, MR Dr. Grün, überwies ihn an die II. Univ.-Augenklinik. Da er auch eine Glaskörperblutung hatte, konnte er dort nicht adäquat mit einer panretinalen Laserkoagulation behandelt werden. Kreisky flog anschließend zur Behandlung nach Boston (USA). Dort wurde er mit höchsten Dosen Acetazolamid behandelt. Das Auge erblindete trotzdem an einem Neovaskularisationsglaukom. Bedauerlicherweise wurde Dr. Kreisky damals nicht an die I. Univ.-Augenklinik zugewiesen, obwohl man ihn hier richtigerweise mit Pars-planaVitrektomie und Netzhautlaser behandeln hätte können. Der Fall ging damals durch die Medien, und die
Berichterstattung warf kein gutes Licht auf die Augenheilkunde in Österreich.

Nach vielen Jahren (2011) stieß der Augenarzt Dr. Hommer (der Nachfolger von Dr. Grün) auf den Krankenakt Kreisky und kontaktierte diesbezüglich den PROFIL-Chefredakteur Dr. Lackner.

Dieser rief mich an, während ich bei einem ophthalmologischen Kongress in den USA weilte, und ich schilderte ihm die Ereignisse um die Augenerkrankung von Dr. Kreisky. Offensichtlich war die Telefonverbindung sehr schlecht, und im PROFIL-Artikel von Dr. Lackner wurden die I. und die II. Univ.-Augenklinik verwechselt [2].

Ein Jahr später, ich war schon Primarius im Donauspital, stieß ich in einer augenärztlichen Boulevardzeitung (Zeitschrift für praktische Augenheilkunde) auf einen Artikel von Herrn Ronald Gerste mit dem Titel: „Dr. Kreiskys Sekundärglaukom“. Der Verfasser dieses Artikels hatte die Tatsachen völlig verdreht und behauptete fälschlicherweise, dass Dr. Kreisky an der I. Augenklinik in Wien behandelt worden sei und deshalb erblindet sei, weil es damals an der I. Univ.-Augenklinik noch keinen „Netzhautlaser“ gegeben hätte [3]. Ich habe damals den Chefredakteur, Reinhard Kaden, angerufen und ihn auf den Fehler aufmerksam gemacht. Er wollte aber keine Richtigstellung schreiben.

Eine weitere Geschichte um eine prominente Persönlichkeit der damaligen Zeit, nämlich Heinz Conrads, betrifft die I. Univ.-Augenklinik: Prof. Heinz Schenk hatte die hochkurzsichtige Gattin des berühmten Schauspielers am grauen Star operiert, so wie damals üblich mit Kryoextraktion und ohne Linsenimplantation. Aufgrund ihrer hohen Kurzsichtigkeit war die Patientin nach der Operation emmetrop und sehr zufrieden. Nach einigen Jahren war Heinz Conrads als Patient an der Reihe, und bei ihm führte die gleiche Operationstechnik zu hoher Unzufriedenheit. Der Patient wurde von seinem Operateur nach Bonn zu Prof. Dardenne geschickt, der Heinz Conrads mit seiner Operation zufriedenstellen konnte. Diese Vorgangsweise war für uns junge Operateure unverständlich, da wir zu dieser Zeit schon extrakapsuläre Kataraktoperationen mit Hinterkammerlinsenimplantation durchführten.

Ich war schon etwa 2 bis 3 Jahre an der I. Univ.Augenklinik tätig, als Herr Prof. Hruby, der sehr unter seiner Parkinson-Erkrankung litt, in Pension ging, und Prof. Schenk wurde Interimsleiter.

Mittlerweile wurde der leider schon verstorbene Dr. Egerer habilitiert und war dann Oberarzt auf der Chefstation. Die neue Leitung der I. Univ.-Augenklinik wurde öffentlich ausgeschrieben, und es gab ca. 15 Bewerbungen aus dem In- und Ausland.

Schließlich wurde der Erstgereihte, Univ.-Prof. Dr. Heinz Freyler, (als Hausbesetzung) neuer Klinikvorstand. Von da an gab es eine rasante Entwicklung auf dem operativen Gebiet. Bald wurden nur mehr extra- 
kapsuläre Kataraktoperationen mit Hinterkammerlinsenimplantation durchgeführt.

Bald kamen die Kapsulorhexis und die Phakoemulsifikation zur Anwendung. Die starren PMMA-Linsen wurden durch faltbare Linsen abgelöst, und schließlich kam es zur Kleinschnittchirurgie.

Ich wurde von Prof. Freyler als Oberarzt auf die „Chefstation“ geholt und führte das Zimmer 7 in Eigenverantwortung, d.h., ich war für die operative Betreuung der dort liegenden Patienten verantwortlich.

Für den Wochenenddienst musste man damals am Samstag um 8:00 Uhr ins Spital kommen und blieb ohne Unterbrechung bis Montagmittag. Ich kann mich noch an einen Wochenenddienst erinnern, an dem ich 5 perforierende Verletzungen hintereinander operierte - nach dem heutigen Arbeitszeitgesetz eine undenkbare Situation. Ich empfand dies damals als „normal“ und habe darunter nicht gelitten. Auch zu Silvester hatte ich oft Nachtdienst, und 2 bis 3 schwere Verletzungen waren das Minimum, das es operativ zu versorgen gab. Zwischen 2 Operationen haben wir um Mitternacht im großen Krankensaal zum Gaudium der Patienten mit den Schwestern den Donauwalzer getanzt und gingen danach wieder in den Operationssaal.

Ich erlebte in den Nachtdiensten viele dramatische und zum Teil auch kuriose Begebenheiten.

Einmal wurde um Mitternacht eine Patientin eingeliefert, deren Ehemann ihr aus Eifersucht beide Augen „ausreißen“ wollte. Beide Augäpfel waren geplatzt und olivenförmig verformt.

Der Augeninhalt war größtenteils ausgetreten. Ich operierte diese Patientin $8 \mathrm{~h}$ lang bis in den Vormittag des nächsten Tages. Ein Auge konnte ich nicht mehr retten, und es ging in Phthisis über, das andere Auge erreichte immerhin einen Visus von 2/60. Einen Tag später las ich in der Zeitung, dass der Ehemann Selbstmord begangen hatte.

Eine andere dramatische Begebenheit handelt von einer 14-jährigen Patientin, die von der Rettung mit einer beidseitigen perforierenden Verletzung eingeliefert wurde. Sie hatte in beiden Hornhäuten einen senkrechten Schnitt von 6 nach 12 Uhr. Auch diese Patientin operierte ich stundenlang.

Nach der Operation schaltete ich die Polizei ein, da ich von einem Fremdverschulden ausging.

Es stellte sich aber heraus, dass die Patientin eine Flasche Nagellack über einer Flamme erhitzt hatte, weil der Verschluss nicht zu öffnen war. Das kleine Glasgefäß explodierte und fügte ihr diese schweren Verletzungen zu. Auch bei dieser Patientin ging ein Auge in der weiteren Folge in Phthise über, das andere Auge erzielte immerhin einen 6-m-Visus.

Ein anderes Mal wurde von der Rettung im Vorfeld eine schwere beidseitige Augenverletzung angekündigt. Ich alarmierte schon die Schwestern im Operationssaal und den Anästhesisten.

Es wurde ein junger Mann auf einer Tragbahre in den Untersuchungsraum gebracht.
Er hatte einen Verband über beiden Augen. Es stellte sich dann heraus, dass er am Gletscher Ski fahren war und sich „nur verblitzt“ hatte. Also eine eher harmlose Angelegenheit.

Prof. Freyler hatte über die Jahre für Diabetiker eine Ambulanz aufgebaut. Diese übertrug er nach seiner Ernennung zum Klinikchef an Dr. Ulrich Klemen. Als dieser Primarius in St. Pölten wurde, übernahm ich dann die Leitung dieser Ambulanz.

Im Bereich der Netzhautchirurgie gab es bereits bei meinem Eintritt in die Klinik die kombinierte Ablatiooperation mit Kryoplexie des Defektes, Aufnähen einer Silikonschaumplombe und transskleraler Subretinaldrainage. Im Laufe der 80er-Jahre wurde dann bei uns an der I. Augenklinik die Silikonfüllung des Glaskörperraums nach Pars-plana-Vitrektomie bei „schwierigen“ Netzhautabhebungen (proliferative Vitreoretinopathie) eingeführt [4].

Im Jahr 1983 wurde ich Oberarzt der Klinik und habilitierte mich mit Arbeiten über die „Arteriitis temporalis Horton“.

Nach der Ernennung von Prof. Freyler zum Klinikchef wurden die Leitungen der einzelnen Stationen neu besetzt: Hans Gnad bekam die Leitung von Station 58, Ulrich Klemen die Leitung der Station 57b, Susanne Binder leitete die Station 19, dort lagen auch die „Schielpatienten“ von Elfriede Stangler-Zuschrott.

In den weiteren Jahren wurde Ulrich Klemen Primarius im LKH St. Pölten und Hans Gnad Primarius im KH Lainz (heute Hietzing). Ich übernahm die Leitung der Station 57b, und Christian Skorpik wurde Leiter der Station 58. Rupert Menapace wurde Oberarzt auf der Chefstation.

Nach dem Abgang von Hans Gnad wurde ich Personalchef der I. Univ.-Augenklinik und war für die Aufnahme vieler neuer Kolleginnen und Kollegen verantwortlich.

Dr. Paul Drobec und Prof. Heinz Freyler schwebte immer ein eigenes wissenschaftliches Journal der Österr. Ophthalmologischen Gesellschaft vor. Im Jahr 1987 war es dann so weit, und das spektrum der augenheilkunde wurde aus der Taufe gehoben.

Im Jahr 1988 wurde die Leitung der Augenabteilung des Donauspitals (SMZ Ost) ausgeschrieben.

Über der Donau sollte ein neues Schwerpunktkrankenhaus entstehen mit einer eigenen Augenabteilung. Die Augenabteilung sollte aus 32 Betten bestehen, einer großen Augenambulanz und 2 Operationssälen. Ich bewarb mich und wurde schon 1988 zum Primarius ernannt, obwohl der Zeitpunkt der Eröffnung der Augenabteilung dann erst der 01.09.1994 war.

Zwischenzeitlich war ich 1991 zum a.o. Univ.-Professor ernannt worden.

Ich erlebte dann im Sommer 1994 noch als Angehöriger der Klinik die Übersiedlung der I. Augenklinik ins „Neue AKH“.

Die Übersiedlung vom AKH in die neu geschaffene Augenabteilung im Donauspital (SMZ Ost) war für alle Beteiligten eine große Herausforderung. Ich musste 
die gesamte medizinische Einrichtung der Ambulanz und der Operationssäle organisieren.

Außerdem musste die Abteilung von Beginn an mit Pflegepersonal und ärztlichem Personal ausgestattet sein. Drei OberärztInnen und eine Fachärztin erklärten sich bereit, mit mir in die Donaustadt zu übersiedeln. Es waren dies Ingrid Kraupp, Alex Salomon, Sascha Rheinberger und Sibylle Stifter. Auch 2 leitende Schwestern (Schwester Kogelmann und Schwester Illetschko) der I. Univ.-Augenklinik übersiedelten mit mir ins Donauspital und hielten mir bis $\mathrm{zu}$ meiner Pensionierung die Treue.

Im 1. Nachtdienst gab es Probleme. Ein Betrunkener mit schwerer Lid- und Tränenwegverletzung wurde eingeliefert. Das Operationsbesteck für Tränenwegverletzungen war noch nicht geliefert worden. Ich fuhr damals ins AKH und borgte mir die Kassette mit dem Besteck für die Operation aus. Das alles geschah um 2:00 Uhr in der Nacht.

Des Weiteren gab es keine Startschwierigkeiten mehr, und wir haben uns alle gut im 22. Bezirk eingelebt.

Jeden Tag kamen immer mehr Patienten in die Augenambulanz, und mit jedem Tag wurden mehr Operationen durchgeführt. Schon nach kurzer Zeit war es mir gelungen, 6 KollegInnen zu „Ablatiooperateuren“ auszubilden. Ich bin sehr stolz darauf, dass im Donauspital Patienten mit Netzhautabhebungen unmittelbar nach der Aufnahme noch in derselben Nacht operiert werden.

Dies ist v. a. bei Patienten mit noch anliegender Makula von großem Vorteil.

22 Jahre lang durfte ich als Vorstand bis zu meiner Pensionierung am 31.07.2016 diese ausgezeichnete Abteilung mit ihrem wunderbaren Team führen.

Während meiner Zeit als Primarius im Donauspital war ich 8 Jahre lang Obmann der österreichischen Augenprimarärzte.

Für 1 Jahr war ich Präsident der Wiener Augenärztlichen Gesellschaft und 9 Jahre lang war ich Obmann der Wiener Primarärzte (alle Fachrichtungen).

Ab dem Jahr 2003 war ich Vorsitzender des fachspezifischen Prüfungsausschusses für das Fach „Augenheilkunde und Optometrie“.

Seit damals war ich für die Prüfungsfragenauswahl von insgesamt 16 Facharztprüfungen verantwortlich. 449 Prüfungskandidaten haben in dieser Zeit die Facharztprüfung abgelegt.

Von 2013 bis 2015 war ich Präsident der Österreichischen Ophthalmologischen Gesellschaft.

Ich habe in meiner Zeit als Augenarzt die Entwicklung der Kataraktchirurgie von der Kryoextraktion der Linse und Korrektur der Fehlsichtigkeit mit Kontaktlinsen oder Starbrille bis zur Phakoemulsifikation der Linse mit Implantation einer faltbaren Hinterkammerlinse („no stich“) miterlebt.

In der Glaukomchirurgie gab es zwar keine so bahnbrechenden Entwicklungen, aber die „Röhrchenimplantationen“ haben sich deutlich verbessert, von der „Cardona-Prothese“ zur „XEN-Implantation“. Am Beginn meiner Laufbahn habe ich noch zahlreiche Zyklodialysen durchgeführt, diese Methode wurde aber heute fast vollständig verlassen.

Ich habe in den letzten 40 Jahren auch die Entwicklung der „refraktiven Chirurgie“ miterlebt.

In den 70er- und 80er-Jahren war die radiäre Keratotomie groß in Mode. Als sich dann Berichte von Hornhautektasien nach radiärer Keratotomie häuften, wurde diese Methode verlassen, und an ihre Stelle trat die „Excimerlaserchirurgie“. Ich habe sehr früh die Vorteile dieser Operationsmethode erkannt und war in Österreich der Erste, der die photoablative refraktive Keratotomie durchführte.

Ich habe im Dezember 1992 das 3. Schwind-LaserKeratom, das weltweit ausgeliefert wurde, erhalten. Seit damals führe ich refraktive Chirurgie mit Folgegeräten erfolgreich durch.

Als ich vor 40 Jahren mit der Augenheilkunde begann, wurden an der I. Univ.-Augenklinik bereits Hornhauttransplantationen durchgeführt. Es gab bereits früh Ansätze für lamellierende Techniken. Die Endotheltransplantation mit ihren verschiedenen Ausführungstechniken ist eine Entwicklung der letzten Jahre (DMEK und DSAEK).

In der Netzhautchirurgie zeichnet sich in letzter Zeit immer mehr eine Abkehr von der Plombenchirurgie und Cerclagetechnik zur primären Vitrektomie ab. Intravitreale Medikamentenapplikationen sind eine Entwicklung der letzten Jahre und waren am Beginn meiner augenärztlichen Laufbahn eine Rarität.

In den 40 Jahren meiner augenärztlichen Tätigkeit war mir immer die positive und persönliche Beziehung zwischen Arzt und Patienten wichtig. Die neueren Entwicklungen, dass wie am Fließband operiert wird (Kataraktchirurgie) und der Patient seinen Operateur gar nicht mehr kennenlernt, sind in meinen Augen nicht positiv zu sehen.

Die Politik mit ihrem überbordenden Spargedanken macht auch vor der Augenheilkunde nicht Halt.

Es droht ein massiver Qualitätsverlust zuungunsten der Patienten, da Wien-weit Nachtdienste reduziert werden sollen. Als Gipfelpunkt der Fehlentwicklungen gibt es den Plan, die Augenabteilung im Donauspital zu schließen.

Man kann nur hoffen, dass auch in weiteren 40 Jahren die augenärztliche Betreuung auf annähernd hohem Niveau wie heute für alle Bevölkerungsschichten gegeben sein wird.

Ich selbst bin weiterhin mit großer Freude in der Ordination und im Operationssaal augenärztlich tätig.

Interessenkonflikt T.M. Radda gibt an, dass kein Interessenkonflikt besteht. 


\section{Literatur}

1. Gnad H, Radda TM, et al. Spätergebnisse der pars plana Vitrektomie bei Diabetikern. Klin Monatsbl Augenheilkd. 1981;179(12):508-11.doi:10.1055/s-2008-1057378.

2. Lackner H. Fatale Nebenwirkungen bei Bruno Kreisky. https://www.profil.at/home/fatale-nebenwirkungenbruno-kreisky-287589. Zugegriffen:09. Jun 2017.
3. Gerste RD. Praktische Augenheilkunde. 2012; 2012(33): 199-2001

4. Gnad H, Radda TM, et al. Silikonölimplantation, erste Erfahrungen mit einer modifizierten Technik. Klin Monatsbl Augenheilkd. 1983;183(11):384-6. doi:10.1055/s-20081054955. 